\title{
Traduire
}

Une autre perspective sur r tr traduction

Revue française de la traduction

$226 \mid 2012$

Face au miroir

\section{Traduction, réalité et fiction dans le désert mauve de Nicole Brossard}

\section{Patricia Godbout}

\section{(2) OpenEdition \\ 1 Journals}

\section{Édition électronique}

URL : http://journals.openedition.org/traduire/140

DOI : $10.4000 /$ traduire. 140

ISSN : 2272-9992

\section{Éditeur}

Société française des traducteurs

\section{Édition imprimée}

Date de publication : 1 janvier 2012

Pagination : 39-44

ISBN : 039-773X

ISSN : 0395-773X

\section{Référence électronique}

Patricia Godbout, «Traduction, réalité et fiction dans le désert mauve de Nicole Brossard », Traduire [En ligne], 226 | 2012, mis en ligne le 03 février 2014, consulté le 10 octobre 2020. URL : http://

journals.openedition.org/traduire/140; DOI : https://doi.org/10.4000/traduire.140 


\title{
Traduction, réalité et fiction dans le désert mauve de Nicole Brossard
}

\author{
Patricia Godbout
}

\section{NOTE DE L'ÉDITEUR}

Version originale française de :

« Übersetzung, Realität und Fiktion : Nicole Brossards Le Désert mauve », trad. Carola

David, dans Klaus Kaindl et Ingrid Kurz dir., Machtlos, selbstlos, meinungslos?

Interdisziplinäre Analysen von ÜbersetzerInnen und DolmetscherInnen in belletristischen

Werken, Vienne, LIT-Verlag, 2010, p. 119-126.

1 Dans un article portant sur «Le traducteur, personnage de fiction», Daniel Simeoni jetait un regard sur des romanciers étrangers contemporains mettant en scène cette figure du passeur qui, invisible hier encore, écrivait-il, « est devenu figure centrale de la création »(2004:24) : cette remarque fait allusion à une évolution du personnage du traducteur dans la fiction contemporaine, évolution que j'ai entrepris d'étudier dans un projet de recherche en cours visant à dresser le portrait du traducteur et de la traductrice tel qu'il se dégage des œuvres littéraires écrites par des auteurs québécois, de langue française et anglaise, depuis 1960. En guise d'entrée en matière, je me propose d'étudier quelques personnages significatifs de traducteurs dans la fiction québécoise avant 1960.

2 Jacques Poulin est un exemple d'auteurs qui ont pratiqué la traduction et créé dans leur fiction des personnages de traducteurs. Les traducteurs fictifs que créent ces auteurs témoignent souvent des questionnements, angoisses et frustrations de l'écrivaintraducteur. Toutefois, dans le cas du roman plus récent de Poulin intitulé La traduction est une histoire d'amour, l'écrivain- traducteur cède le pas à l'écrivain qui sait apprécier tout le soin mis par sa traductrice (en l'occurrence la traductrice littéraire canadienne bien connue Sheila Fischman) à rendre ses romans en anglais. Pour son roman Le Désert mauve, Nicole Brossard a affirmé s'être inspirée entre autres, des échanges qu'elle avait 
eus avec Patricia Claxton, traductrice vers l'anglais d'un de ses romans précédents intitulé French Kiss. Pour faire écho au titre du roman de Poulin, on peut dire que Brossard a créé dans Le Désert mauve une traductrice, nommée Maude Laures, qui vit une histoire d'amour avec le roman qu'elle a entrepris de traduire, celui de Laure Angstelle. Comme le note Anne-Marie Wheeler: «Non seulement l'auteure et la traductrice partagent l'amour des mots, mais Maude Laures tombe amoureuse de l'usage particulier qu'en fait Laure Angstelle » (Wheeler $2003: 445$ ).

3 J'aimerais justement me pencher sur cette œuvre qui a joué un rôle indéniable dans la redéfinition du paradigme de la traduction dans les lettres québécoises. J'estime en effet que Le Désert mauve a contribué à redéfinir la traduction non plus seulement en termes linéaires, mais comme entrelacement du corps du texte à traduire et du corps du texte traduit.

\section{Un livre à traduire}

4 Rappelons quelles sont les parties principales de ce livre de Nicole Brossard: il y a d'abord le roman «Le Désert mauve ", par Laure Angstelle (qu'on dit être publié aux Éditions de l'Arroyo), roman qui a sa pagination propre, le livre à traduire (le projet de Maude Laures) et enfin, la « traduction » elle-même, « Mauve, l'horizon ».

Dans ce roman de Laure Angstelle écrit à la première personne, la narratrice, Mélanie, quinze ans, a compris que "[l]a beauté est avant la réalité »(p.36). Elle enjoint sa lectrice de ne pas laisser la réalité la divertir d'elle-même, de ne pas laisser celle-ci « empiéter sur les êtres comme une distorsion tragique de la beauté » (p. 36), de ne pas céder « devant l'aura tragique » (p. 40). Cette lectrice et traductrice en puissance a tôt fait de comprendre que la «beauté antérieure »est aussi donnée comme étant celle du livre à traduire : « Vous m'êtes antérieure » (p. 140), dit la traductrice à l'auteure.

Mélanie roule à vive allure dans le désert, dans la « Météor » de sa mère. La lectrice doit monter, elle aussi, à bord de la Météor, où elle expérimentera la peur, la soif, le désir, le mauve. «Comment pourrais-je déserter Mélanie?» (p.154), se demande-t-elle - à l'instar de la narratrice, auteure, traductrice.

$7 \quad$ Le sens des mots est sans cesse interrogé : celui de désert en particulier. Ce dernier est à la fois vrai, dangereux (p. 30) - là où l'écriture se perd - et à la fois contrepoids à la réalité, espace permettant les longueurs dans la pensée (p. 37) ; il est aussi chaleur qui rend les êtres vivants (p. 37). En ce cas, « la chaleur» de l'écriture redonne vie au mot "vivant» lui-même - grâce à cette articulation nouvelle qui en fait un attribut du complément d'objet.

Avec la deuxième section du roman intitulée "Un livre à traduire ", «l'histoire » se poursuit sous la forme de réflexions sur l'écriture et sur la lecture aussi bien que sur la traduction, opérations toutes englobées dans «l'acte ininterrompu de l'interprétation » (p. 58). Maude Laures, la traductrice, n'en a pas moins saisi qu'elle est la troisième personne (après l'auteure et la lectrice). Elle sait que traduire arrive au terme d'un processus : «nommer, [...] converser longuement [...] jusqu'à [...] la [...] tentation de traduire » (p. 56). Il y a la nécessaire séduction exercée par le livre à traduire. « Maude Laures s'était laissé séduire, ravaler par sa lecture » (p. 59), écrit Brossard. Ravaler, au sens de ramener à la beauté. Traduire, c'est ainsi être un faire-valoir, un faire-avaler, un faire-ravaler. 
Studieuse, Maude Laures s'applique à colliger des indices de son désir du désert qui la rendra diserte à son tour. Il lui faudra trouver le chemin qui va de Maude Laures au monde de Laure, il lui faudra monter à bord de la Météor. La rapidité sera nécessaire pour que - comme l'écrit Brossard en prenant ici un accent bermanien (je pense ici plus particulièrement aux préoccupations sourcières de Berman telles qu'exprimées dans La traduction et la lettre ou l'auberge du lointain, 1999) - Maude Laures « recouvre chaque mot d'un autre mot sans que le premier ne sombre dans l'oubli » (p. 65).

Il lui faudra aller au-delà de la réciprocité, de l'aller-retour, de la lecture à rebours - qui est de toute manière chose impossible. Car si « Mauve, l'horizon » force un retour sur le "premier »texte ("Le Désert mauve»), l'«Angle» de lecture sera nécessairement différent. La lectrice du livre traduit advenu se trouve ainsi, bon gré mal gré devant deux états d'un même manuscrit - deux états proches de l'Arizona.

11 Le risque qu'il y a à entrer dans le désert est grand. La traductrice a la peur au corps. Elle est tenaillée par la peur panique, l'angoisse, de se substituer à l'auteure du livre à traduire. Le mot de Maude Laures avalera-t-il le mot de Laure Angstelle? Ainsi, la peur est présente aussi bien dans l'écrire que dans le traduire. Comme le note très justement Anne-Marie Wheeler: «La panique du traducteur confronté à l'acte d'interpréter une langue dans une autre devient le prolongement de la crainte ressentie par l'auteure transposant d'abord sa peur » (2003: 444).

12 Mais dans les deux cas, c'est une peur porteuse d'un livre à venir (Blanchot). La traductrice tremble d'être la mère porteuse du livre d'une autre. Mais ce qui pouvait se lire au départ comme un rimbaldien dérèglement de tous les sens (p. 58) devient ici, dans le Mauve, un exercice en souplesse qui nettoie la peur (p.59). La méthode de travail décrite vaut d'ailleurs pour l'auteure comme pour la traductrice : il faut faire des fiches, annoter les pages selon un code de couleur (noir incompréhensible, jaune familier, etc., p. 169) - qui fait écho au sonnet rimbaldien des "Voyelles». Il faut isoler la réalité, l'insonoriser (p. 157), braquer ses pensées sur l'horizon, afin que la beauté qui précède procède (p.161). Être en voie de traduction, c'est devenir cette passagère assoupie qui se voit en rêve au volant de la Météor.

On aura compris qu'on ne s'encombre pas longtemps de préséances dans le désert. Certes, on a recouru aux formules d'usage pour reconnaître que si «[t]out avait [...] été possible dans la langue de l'auteure ", la traductrice devrait, dans la sienne, s'armer de beaucoup de patience (p. 59). On a même affirmé haut et fort que la traductrice n'avait aucun droit, ayant «choisi la tâche difficile de lire à rebours » dans sa langue ce qui, dans celle de l'auteure, « coule de source » (p. 142f.).

Mais la primauté omnisciente de l'auteure a tôt fait d'être remise en question : « Peutêtre [Laure Angstelle] n'avait-elle jamais connu le désert»(p.90), soupçonne la traductrice Maude Laures. De sorte qu'en dépit de l'antériorité de la beauté, outre la linéarité de l'avant et de l'après, une autre figure se dessine, se profile : c'est celle du zeugme, de l'accouplement des mots avec économie de moyens. On en trouve un exemple à la fin du roman, lorsque dans « Mauve, l'horizon » Mélanie affirme, sous la plume de Maude Laures, qu'elle n'a «jamais cessé de croiser le fer et le sentiment » (p. 191).

15 Ce zeugme serait emblématique, à mon avis, de ces «étranges accouplements » entre livre à traduire et traduction à finir. Il serait à l'image de cette «forme nouée d'une 
double présence » (p. 190), celle de deux femmes ensemble : la mère de Mélanie assise sur les genoux de son amie Lorna, dans le regard de la narratrice et le nôtre.

\section{Tensions entre réalité et fiction}

16 La traductrice fictive dans cette œuvre est intéressante à plus d'un titre, en raison tout particulièrement de sa façon d'interroger le rapport entre réalité et fiction. Selon Beverley Curran, la lecture que fait Maude Laure du roman de Laure Angstelle est précisément l'occasion d'un dialogue entre réalité et fiction (cf. 2000 : 173). En cours de travail, la traductrice en vient en effet à se demander comment aurait pu être évitée la fin brutale que connaît dans le roman de Laure Angstelle le personnage d'Angela Parkins. Cette interrogation débouche sur une réflexion sur le langage. «Maude Laures veut débattre de la mort d'Angela Parkins, mais pas du point de vue d'un lecteur ", souligne Curran $(2000: 174)$. Elle deviendrait l'un des personnages du roman de Laure Angstelle, elle deviendrait Angela Parkins. Mais l'auteure la ramène à la réalité de l'homme qui tue parce qu'il ne peut supporter l'amour lesbien. Il n'y a donc pas, dans le récit lui-même, de sentiers qui bifurquent à la manière de Borges. Le texte n'en crée pas moins un univers fictif et langagier s'inscrivant contre "cet homme [qui] existe " (p. 142) « dans les personnages d'une femme écrivant, d'une femme lisant et de femmes parlant, leur présence dans la fiction produisant un effet chaque fois que ce livre est entre les mains d'une lectrice » $(2000: 176)$. La traduction du Désert mauve en plusieurs langues, de surcroît par des femmes dans le cas de l'anglais et de l'allemand, prolonge cette transmission.

17 Comme le précise Wheeler: «Pour N. Brossard, l'une des énigmes majeures de l'écriture réside dans le point où langue et réalité s'opposent, comme cela est mis en évidence par le processus de traduction » (2003 : 440). Ainsi, N. Brossard prend-elle le parti d'investir la langue, avec toutes les difficultés et même la violence qui lui sont inhérentes. Elle invite ses lectrices à prendre conscience de la puissance que recèle celle-ci (2003 : 443). Le thème de la traduction devient donc pour N. Brossard l'outil métaphorique qui lui permet d'explorer le rapport des femmes à la langue, de mettre en relief la difficile prise de parole des femmes dans l'espace- temps du langage.

Cette tension se trouve répercutée d'une manière différente dans une autre œuvre de fiction québécoise mettant en scène une traductrice : il s'agit du roman Traductrice de sentiments d'Hélène Rioux (1995) dans lequel la narratrice souffre d'avoir à rendre en français la parole d'un tueur en série dont elle traduit l'autobiographie. Chez $\mathrm{N}$. Brossard, la traduction est employée plus métaphoriquement, pour ainsi dire, à cause de la tonalité poétique du roman et de la réflexion induite par la traduction du français au français qui s'opère dans le roman. Mais le fait que $\mathrm{H}$. Rioux donne la parole à une traductrice aux prises avec le discours d'un homme violent n'en est pas moins une façon d'amener un questionnement sur le rapport de la femme au langage. On pourrait également évoquer la difficulté d'avoir à traduire certaines poésies misogynes dont fait état, dans Re-Belle et Infidèle (1991), Suzanne de Lotbinière-Harwood, qui est par ailleurs la traductrice vers l'anglais du Désert mauve de Nicole Brossard.

19 En outre, dans le contexte linguistique propre au Québec, la traduction du français au français sur laquelle repose l'œuvre s'avère digne d'intérêt. Loin d'être une stratégie pour refuser l'altérité de la langue anglaise comme on pourrait le croire de prime abord, ce choix d'écriture permet au contraire de dépasser l'habituelle tension entre 
l'anglais et le français pour explorer l'hétérogénéité propre au français (cf. Suchet 2009). Ce serait également une manière de souligner la multiplicité des paroles féminines.

Ainsi, bien que la traduction intralinguale soit, on le sait, l'un des trois types de traduction définis par Roman Jakobson dans ses Essais de linguistique générale (1963: 79), l'emploi potentiellement discutable du mot «traduction» pour désigner la reformulation en français qu'effectue Maude Laures du roman écrit dans cette « même » langue par Laure Angstelle n'est pas la seule manifestation du caractère fictif de la traduction dans Le Désert mauve. En effet, du fait de la connotation négative attachée, tout au long du roman, au mot "réalité ", une sorte d'alliance se noue dans l'œuvre entre traduction et fiction, ces deux termes se liguant pour créer un espace d'écriture habitable qui en viendra progressivement à faire partie de la "réalité ». Notons d'ailleurs que la réduction délibérée - ou le brouillage - de l'illusion référentielle fait partie des pratiques avouées d'écriture de Nicole Brossard (Paranhos $2007: 13)$.

Un rapprochement s'opère ainsi entre la traduction et la parole féminine, lesquels sont traditionnellement, rappelons-le, deux discours ancillaires. La crainte ancestrale éprouvée à l'égard du traducteur - le plus souvent attribuée au potentiel de traîtrise de son activité - est mise en parallèle avec celle que génère la parole féminine, souvent associée à la séduction, mot qui, au sens strict, signifie détournement du droit chemin. Toutefois, dans maintes réflexions traductologiques, la place subalterne de la traduction de même que le rapport hiérarchique auteur- traducteur sont mis en cause ; la traduction représente au contraire le potentiel d'ouverture de l'œuvre tout en constituant le lieu par excellence de réflexion sur la langue (Benjamin 2000). On observe un développement réflexif parallèle dans les études féministes, où il y a prise de conscience du pouvoir de la langue et de l'importance, pour les femmes, d'investir cet espace. Le fait que ces deux champs de réflexion coïncident dans Le Désert mauve en décuple indubitablement la portée.

\section{BIBLIOGRAPHIE}

BENJAMIN, Walter 2000, « La tâche du traducteur », Euvres. Trad. M. de Gandillac, R. Rochlitz et P. Rusch, Paris, Gallimard, p. 244-262.

BERMAN, Antoine 1999, La traduction et la lettre ou l'auberge du lointain, Paris, Gallimard.

BROSSARD, Nicole 1987, Le Désert mauve, Montréal, L'Hexagone. Réédition 1993 et 2010 (coll. Typo). Trad. anglaise Susanne de Lotbinière-Harwood, Mauve Desert, Toronto, Coach House Press, 1990. Trad. allemande Traude Bürhman, Die malvenfarbene Wüste, Munich, Frauenoffensive, 1989.

1974, French Kiss : étreinte-exploration, Montréal, Éditions du Jour. Trad, anglaise Patricia Claxton, French Kiss, or a Pang's Progress, Toronto, Coach House, 1986.

CURRAN, Beverley 2000, « Reading Us Into the Page Ahead : Translation as Narrative Strategy in Daphne Marlatt's Ana Historic and Nicole Brossard's Le désert mauve », dans Lieven d'Hulst et John 
Milton (dir.), Reconstructing Cultural Memory: Translation, Scripts, Literacy, Amsterdam et Atlanta, Rodopi, p. 165-177.

DE LOTBINIÈRE-HARWOOD, Suzanne, 1991, Re-belle et infidèle : la traduction comme pratique de réécriture au féminin / The Body Bilingual : Translation as a Re-Writing in the Feminine, Toronto et Montréal, Women's Press et Éditions du Remue-Ménage.

JAKOBSON, Roman 1963, « Aspects linguistiques de la traduction », dans Essais de linguistique générale, trad. Nicolas Ruwet, Paris, Minuit, p. 78-86.

PARANHOS, Ana Lúcia S. 2007, «Le désert mauve : l'enjeu de la traduction vu par Nicole Brossard », Interfaces Brasil/Canada, Rio Grande, $\mathrm{n}^{\circ}$ 7, p. 9-32.

RIOUX, Hélène 1995, Traductrice de sentiments, Montréal, xYZ, coll. « Romanichels ». Réédition 2008 (coll. « Romanichels poche»).

SIMEONI, Daniel 2004, « Le traducteur, personnage de fiction », Spirale, juillet-août, p. 24-25.

SUCHET, Myriam 2009, "Traduire du français au français : proposition pour un "comparatisme différentiel" ", NT2. Nouvelles technologies, nouvelles textualités, cahier virtuel du Laboratoire de recherche sur les œuvres hypermédiatiques, Université du Québec à Montréal, septembre, $\mathrm{n}^{\circ} 4$, article 05. (http://nt2.uqam.ca/recherches/cahier/article/)

WHEELER, Anne-Marie 2003, «Issues Of Translation In the Works Of Nicole Brossard », The Yale Journal of Criticism, vol. 16, $\mathrm{n}^{\circ}$ 2, automne, p. 425-454.

\section{AUTEUR}

\section{PATRICIA GODBOUT}

Patricia.Godbout@USherbrooke.ca

Patricia Godbout est professeure de traduction à l'Université de Sherbrooke, au Québec. Elle a publié un livre et plusieurs articles sur l'histoire de la traduction littéraire au Canada. Elle prépare présentement une monographie sur les personnages de traducteurs et d'interprètes dans les romans québécois. Elle est également traductrice, principalement d'ouvrages de critique littéraire. 